\title{
Charge induced stability of water droplets in subsaturated environment
}

\author{
J. K. Nielsen ${ }^{1}$, C. Maus ${ }^{2}$, D. Rzesanke ${ }^{3}$, and T. Leisner ${ }^{3}$ \\ ${ }^{1}$ Danish Meterological Institute, Lyngbyvej 100, 2100 Kbh Ø, Denmark \\ ${ }^{2}$ Technical University Ilmenau (TUI), Institute for Physics, Postfach 100565, 98684 Ilmenau, Germany \\ ${ }^{3}$ Karlsruhe Institute of Technology (KIT), Institute for Meteorology and Climate Research, Hermann-von-Helmholtz-Platz 1, \\ 76344 Eggenstein-Leopoldshafen, Germany
}

Received: 1 October 2010 - Published in Atmos. Chem. Phys. Discuss.: 2 November 2010

Revised: 22 February 2011 - Accepted: 28 February 2011 - Published: 7 March 2011

\begin{abstract}
Atmospheric liquid and solid water particles are stabilized if they are coated with either negative or positive electric charge. The surface charge causes an increase of the partial pressure of water vapour close to the surface of each particle, effectively allowing the particles to remain in their condensed phase even if the environmental relative humidity drops below unity. The theory, briefly presented in this paper, predicts a zero parameter relation between surface charge density and water vapour pressure. This relation was tested in a series of Electrodynamic Balance experiments. The measurements were performed by stabilizing charged droplets of pure water near an ice-surface. We observed a divergence in radius as the temperature approached the freezing point from below. We find that the measurements confirm the theory within the experimental uncertainty. In some cases this generally overlooked effect may have impact on cloud processes and on results produced by Electrodynamic Balance experiments.
\end{abstract}

\section{Introduction}

Recently a few field measurements performed at different locations in the tropics has lead to observations of ice particles in the lowest stratosphere (Nielsen et al., 2007; Chaboureau et al., 2007; de Reus et al., 2008; Corti et al., 2008). Occurrence of ice particles in the stratosphere is somewhat unexpected since the stratosphere is generally subsaturated with respect to ice. This is indeed the case in the measurements of Khaykin et al. (2009). These measurements must prompt speculations about which mechanisms could prevent ice from

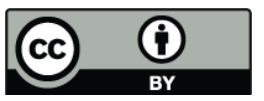

Correspondence to: J. K. Nielsen (jkn@dmi.dk) sublimating in the lowest stratosphere. A possible explanation could be influence of electric charge which may be abundant in some areas of interest. The idea is basically that charges sitting on particles perturb the gas phase water dipoles locally, by attracting water molecules close to the ice surface. While the sparseness of in situ observations leaves some room for disputing this concept we pursue the question from a purely experimental point of view in this paper. Our approach is to measure the size of an evaporating supercooled water droplet, with diameter in the range 10-100 $\mu \mathrm{m}$, trapped in an Electrodynamic Balance (EDB). See Achtzehn et al. (2005) and Davis (2005) for details about the EDB. During the experiment the EDB chamber is kept in a subsaturated state, i.e. the water vapour partial pressure is kept below the saturation water vapour partial pressure over a plane liquid surface. The theory of gas phase water surrounding a charged droplet is equivalent to the theory of gas phase water surrounding a charged ice particle. So if we are able to observe that charged water droplets in a subsaturated environment are stabilized according to the theory, we take that as proof of concept. These laboratory experiments will allow us to conclude whether or not stratospheric ice particles will be stabilized if charge is present on their surface. The question of whether sufficient charge sometimes is present on stratospheric ice particles cannot be answered from these laboratory experiments, and that question is as such not addressed in this paper.

\section{Theory of charged hydrometeors}

The thermodynamic effects of surface charge on water droplets has historically been addressed a few times in literature (Rusanov, 1979; Thomson and Thomson, 1928; Cohen et al., 1987). The theoretical treatment has been given

Published by Copernicus Publications on behalf of the European Geosciences Union. 
in terms of a reduction of the Kelvin effect, i.e. essentially a reduction of surface tension $\sigma_{\mathrm{s}}$ caused by presence electric charges. This leads, in principle, to a relative decrease of the water vapor pressure e at the surface of the particle. Not until Lapshin et al. (2002) was it recognized that the interaction between gas-phase water dipoles and the charged droplet actually overrules the surface tension effect in many realistic applications. Lapshin et al. derived the expression for the charge dipole interaction and focussed on the limit where $k_{\mathrm{B}} T>>\sigma_{\mathrm{q}} d / \varepsilon_{0}$, here $k_{\mathrm{B}}$ is Boltzmann's constant, $T$ temperature, $\sigma_{\mathrm{q}}$ surface charge density, $d$ the water dipole moment and $\varepsilon_{0}$ the vacuum permittivity. In that limit it turns out that the logarithm of the relative increase of surface partial water vapor pressure $\ln \mathrm{e} / \mathrm{e}_{0}$ follows a $\sigma_{\mathrm{q}}^{2}$ law. Physically it corresponds to the situation where the electric field is so weak that the dipoles can take any orientation in space, with a statistical preference for alignment with the electric field, which would be the case in many applications. However, there are also cases, for instance in applied EDB experiments, where the electric field close to the charged droplet is strong enough to fix the water molecules orientation in space, i.e. $k_{\mathrm{B}} T<<\sigma_{\mathrm{q}} d / \varepsilon_{0}$. In the present study $k_{\mathrm{B}} T /\left(\sigma_{\mathrm{q}} d / \varepsilon_{0}\right)$ is in the order of $10^{-6}$, so in the derivation below we safely assume that water molecules are aligned with the electric field. It is not necessary to consider the kinetic effects of this reduction of degrees of freedom, since the dynamical process of evaporation happens to be based on the (macroscopic) transport properties. In effect this means that no matter which physical processes are in effect close to the surface and inside a droplet, it is still the macroscopic laws of gas and heat diffusion far from the droplet that control the speed of condensation and evaporation. Other effects to be considered, but still negligible in this context, are the additional polarization of the water molecules induced by the electric field and the Kelvin effect (Pruppacher and Klett, 1997). The polarization of water molecules turns out to be completely irrelevant here, because very strong electric fields are required for that effect to have an influence. The Kelvin effect is at least two orders of magnitude from being in effect, and it will be discussed briefly after the derivation below.

\subsection{Equilibrium}

The water vapour pressure e at distance $r=|\boldsymbol{r}|$ from the centre of an equilibrated charged spherical particle may be calculated by requiring that the chemical potential $\mu$ of a gas phase water molecule located close to the charged particle

$\mu_{\mathrm{gas}}(r)=-\frac{q \boldsymbol{r} \cdot \boldsymbol{p}}{4 \pi \varepsilon_{0} r^{3}}+\mu_{0}(T)+k_{\mathrm{B}} T \ln (\mathrm{e}(r))$

is constant in space. Here $q=z e_{u}$ is the electric charge, $\boldsymbol{p}$ the water dipole moment, $\varepsilon_{0}$ the vacuum dielectric constant and $k_{\mathrm{B}}$ Boltzmann's constant. The equilibrium requirement

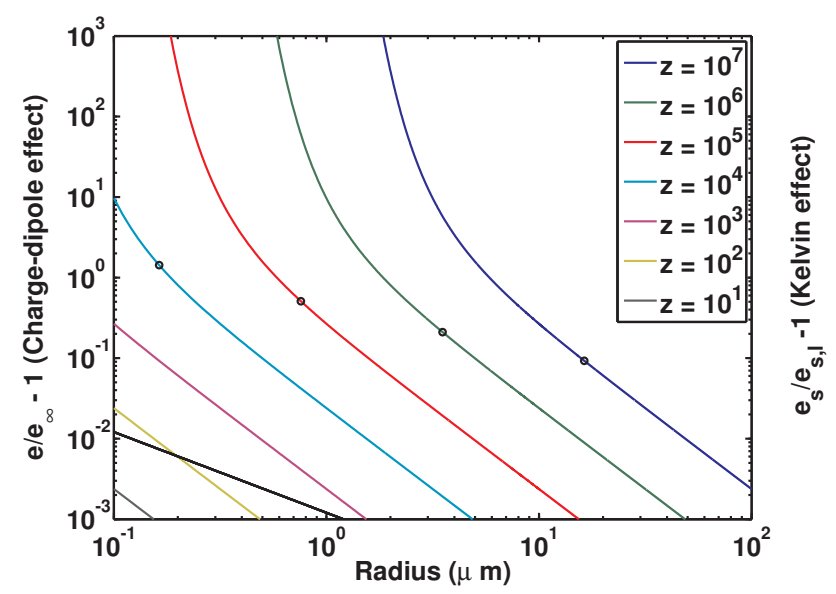

Fig. 1. Relative increase of partial pressure of water (left axis) as function of distance from the centre of a spheric charged hydrometeor for different unit charge numbers, at $T=273 \mathrm{~K}$. The unit charge $e_{u}$ equals $1.602 \mathrm{e}-19 \mathrm{C}$. The circles denote the radius of Rayleigh instability (see text) for a given charge. The black line shows the relative increase of saturation water vapor pressure (right axis) over a droplet due to surface tension, i.e. the Kelvin effect. Note that for a given particle size the Kelvin effect causes the environmental saturation pressure to rise, while the charge effect causes the environmental saturation pressure to fall. See explanation in the text.

$\frac{d \mu_{\mathrm{gas}}}{d r}=0$ leads to an expression for the partial pressure of water close to the particle

$e(r)=e_{\infty} \exp \left(\frac{|q \boldsymbol{p}|}{4 \pi r^{2} \varepsilon_{0} k_{B} T}\right)$

where $e_{\infty}$ is the ambient partial pressure. This relation is plotted in Fig. 1 in terms of relative increase of $e(r)$ for different ice particle radii. Also shown in Fig. 1 is the so called Rayleigh instability radius $r_{\mathrm{R}}=\left(\frac{q^{2}}{64 \pi^{2} \epsilon_{0} \sigma_{\mathrm{s}}}\right)^{1 / 3}$, below which the Coulomb interaction dominates the mechanical stabilization caused by the surface energy $\left(\sigma_{\mathrm{s}}\right.$ is the surface energy density) and the droplet breaks up (Rayleigh, 1882; Duft et al., 2003). The black line in Fig. 1 shows the increase of water vapour saturation pressure, $\mathrm{e}_{\mathrm{s}} / \mathrm{e}_{\mathrm{s}, 1}-1$, caused by surface tension of an uncharged droplet (the Kelvin effect). For droplet radii above $1 \mu \mathrm{m}$ we can safely assume that the surface energy does not influence the saturation water vapour pressure. Note that the two effects work in very different and opposite ways. Where the Kelvin effect increases the saturation pressure of water on the particle surface and through out the gas phase, the charge dipole effect conserves the equilibrium pressure of the droplet surface, while suppressing the partial pressure of water in the gas phase far from the charged hydrometeor. This is because the charge dipole interaction maintains a water concentration gradient in the gas phase close to the charged hydrometeor. In equilibrium the vapour pressure at the surface $\mathrm{e}(r)$ is equal to the saturation 
pressure over an uncharged liquid water surface $\mathrm{e}_{\mathrm{s}, 1}$, so the relation (Eq. 2) may be viewed as an depression of the environmental saturation pressure $e_{\mathrm{s}, \infty}$ above a charged surface as a function of surface charge density $\sigma_{\mathrm{q}}$.

$\mathrm{e}_{\mathrm{s}, \infty}=\mathrm{e}_{\mathrm{s}, 1} \exp \left(-\frac{\left|\boldsymbol{p} \sigma_{\mathrm{q}}\right|}{\varepsilon_{0} k_{\mathrm{B}} T}\right)$.

Physically this means that one would expect to observe a depression of the relative humidity $\mathrm{RH}_{\mathrm{w}}$ to a value below unity in an "equilibrated" charged cloud. Here "equilibrated" means that the system is in thermodynamic equilibrium under the constraint imposed by presence of surface charge, but possibly still participating in mechanical processes like coalescence and sedimentation. $\mathrm{RH}_{\mathrm{w}}$ can be expressed

$\mathrm{RH}_{\mathrm{w}}=\exp \left(-\frac{\left|\boldsymbol{p} \sigma_{\mathrm{q}}\right|}{\varepsilon_{0} k_{\mathrm{B}} T}\right)$.

In equilibrium the droplet size distribution will be determined by the charge of the single droplets, since $\sigma_{\mathrm{q}}$ has to be the same on all droplets. If for instance a droplet had a lower surface charge density than the surrounding droplets it would be out of equilibrium and start to shrink until its surface charge density had equilibrated with the surrounding droplets. If one prefers, Eq. (4) may be viewed as an additional term in the water activity (see e.g., Koop et al., 2000).

\subsection{Non-equilibrium}

The condensation/evaporation of a droplet of radius 1-50 $\mu \mathrm{m}$ in an atmosphere of relative humidity $\mathrm{RH}_{\mathrm{w}}$ may be treated as a macroscopic diffusion problem, i.e. the mass flux to a droplet of radius $r$ may be calculated from a steady state solution to the diffusion equation. The problem has been solved in Pruppacher and Klett (1997, Eq. 13-28), and that solution includes specifically the effects of gas diffusion, and heat diffusion which turns out to be essential. Here, in Eq. (5), we extend the Pruppacher and Klett (1997) formula with a term accounting for the charge effect. While the charge changes the ambient equilibrium water vapour partial pressure in the particle's surroundings, the surface of the particle always obeys the boundary condition $\mathrm{e}(r)=\mathrm{e}_{\mathrm{S}}(T(r))$. This leads to the following differential equation for the particle radius. Note that symbols in Eq. (5) are defined in the Glossary.

$r \frac{d r}{d t}=\frac{\left(1+U_{d}\right) \mathrm{RH}_{\mathrm{w}}-1}{\frac{\rho_{\mathrm{w}} R T_{\infty}}{\mathrm{e}_{\mathrm{s}, \mathrm{I}}\left(\mathrm{T}_{\infty}\right) \mathrm{D}^{*} \mathrm{M}_{\mathrm{w}}}+\frac{L \rho_{\mathrm{w}}}{k_{\mathrm{a}}^{*} T_{\infty}}\left(\frac{L M_{\mathrm{w}}}{T R}-1\right)}$

where

$U_{d}=\frac{|q \boldsymbol{p}|}{4 \pi \varepsilon_{0} k_{\mathrm{B}} T_{\infty} r^{2}}$

\section{Experimental setup}

We now test the validity of the charge stabilization theory in a series of EDB experiments. In order to enable a situation where a hydrometeor is exposed to a well defined partial water vapor pressure below the saturation point, we use the following setup. The EDB chamber is prepared with an ice surface on the chamber wall which is maintained at the same temperature as the captured charged particle. See Fig. 2 for an illustration of the EDB-cell. The ice on the cell walls serves as a buffer to ensure that the $\mathrm{RH}$ is close to $\mathrm{e}_{\mathrm{s}, \mathrm{i}} / \mathrm{e}_{\mathrm{s}, \mathrm{l}}$. However, as it turns out that there are small temperature variations $(\Delta \mathrm{T}<0.1 \mathrm{~K})$ in the cell there may be a slight difference between the RH in the center and the RH on the wall, and therefore we have to estimate the true RH from the evaporation velocity. For these experiments we used NANOpure $(\mathrm{tm})$ water, $>200 \mathrm{~nm}$ filtrated, with a resistivity greater than $18.2 \mathrm{M} \Omega \mathrm{cm}$. Even when the concentration of possible ionic impurities is increased by a factor of 1000 during the evaporation process, their mole fraction is kept below $10^{-4}$. A liquid water charged particle kept in the trap will be subject to a water vapor partial pressure which is in fact the saturation water vapor pressure above ice $\mathrm{e}_{\mathrm{i}}$ i.e. always below saturation pressure of liquid water $\mathrm{e}_{1}$. Under these special conditions the liquid droplet terminal radius $r_{\mathrm{q}}$ is ideally a function of only temperature and charge:

$r^{2}=\frac{|\boldsymbol{p} q|}{4 \pi \epsilon_{0} k_{\mathrm{B}} T \ln \frac{\mathrm{e}_{\mathrm{s}, \mathrm{l}}}{\mathrm{e}_{\mathrm{s}, \mathrm{i}}}}$.

This dependency is shown in a phase diagram in Fig. 3. In the temperature range between $-15^{\circ} \mathrm{C}$ and $0{ }^{\circ} \mathrm{C}$ the predicted $r_{q}$ can reach high measurable values without reaching the Rayleigh stability limit. Generally the particle charge and chamber humidity may be controlled to some extent, but both parameters have to be determined indirectly. The initial charge is calculated from the DC bias field required to balance the particle in the gravitational field, the mass being determined from the measured particle radius. The particle charge is unchanged once the droplet has been injected into the chamber. However, in cases where the droplets reach the Rayleigh instability limit detectable bursts of charge will escape the droplet (Duft et al., 2003), and the electric charge has to be reevaluated. The relative uncertainty on the charge becomes larger in such cases. The temperature is not completely homogeneous throughout the chamber and this results in uncertainty on the humidity, which again causes a considerable scatter on the terminal radius of the droplet. Considering the "charged droplet over ice" relation (Eq. 7) one sees that as the system approaches the triple point, the term $\ln \frac{\mathrm{e}_{\mathrm{s}, \mathrm{l}}}{\mathrm{e}_{\mathrm{s}, \mathrm{i}}}$ becomes "critical" because it is essentially a difference between two almost identical properties. The terminal radius becomes very sensitive to small temperature variations, which leads to a lot of experimental noise. During a single experiment which only lasts a few hundred seconds, the terminal area practically does not drift, which means that resulting RH does not drift either. But between experiments the temperature instability may cause an $\mathrm{RH}$ variation of around $1 \%$. Therefore we choose to determine the partial pressure of water vapour in the trap saddle point from the initial slope 


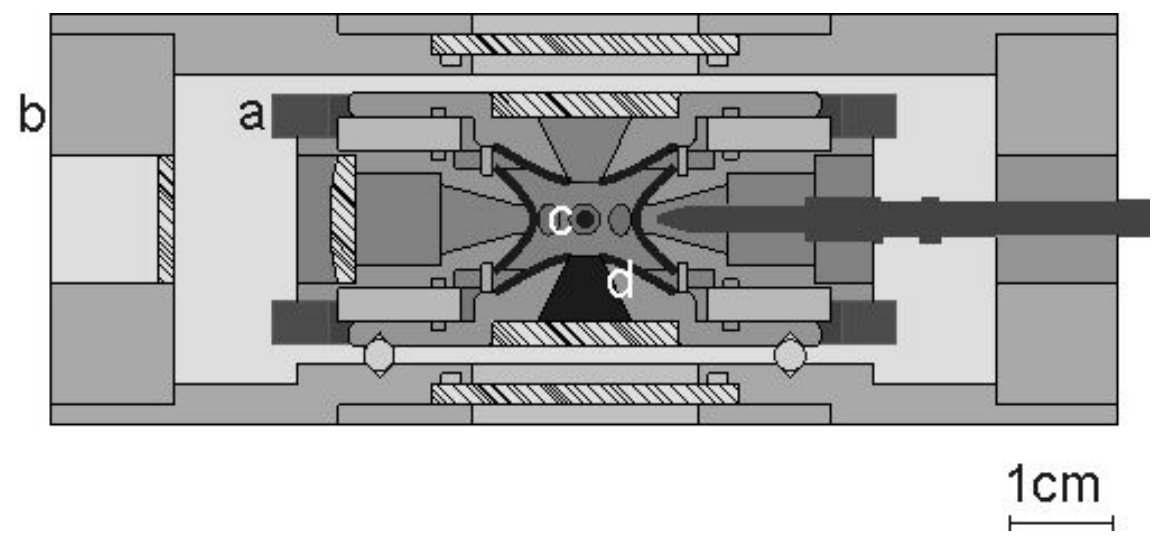

Fig. 2. Cross-section of the temperature controlled levitator (a) inside a vacuum chamber (b). The microdroplet (c) is centered amidst the ice covered electrodes (d) and above an ice reservoir. From the right hand side droplets will be injected and there is also optical access in axial an radial direction.

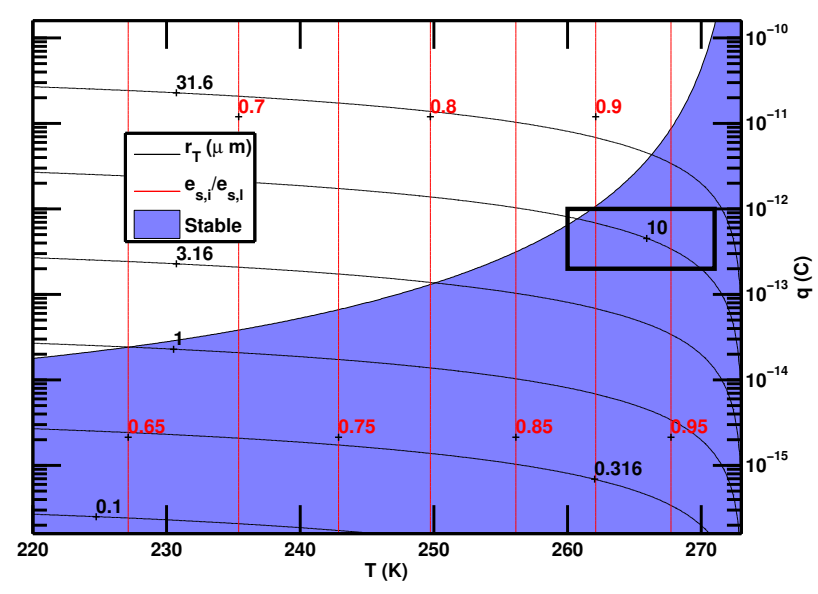

Fig. 3. Terminal radius $(\mu \mathrm{m})$ for a charged droplet above an icesurface, shown as black contours. The red lines show the ratio between ice saturation pressure and water saturation pressure, which only depends on temperature. The colored area shows where the droplet charge is below the Rayleigh instability limit, and the rectangle roughly shows the part of the phase diagram that we can access in these experiments.

of the evaporation curve, by use of Eq. (5). Note that since the charge is determined from the droplet radius and the DCfield, the relative humidity is the only unknown variable in Eq. (5), hence we do in effect have independent measurements of charge and relative humidity for each droplet.

\section{Results}

A series of 163 experiments was performed at two different temperatures $(268.2 \mathrm{~K}$ and $270.2 \mathrm{~K})$. In each experiment a new charged droplet of otherwise clean water was captured in the trap. The droplets immediately started to evaporate after they had been captured. In Fig. 4 we show an evaporation curve (surface area versus time). The diameter is measured directly from camera images obtained at $5 \mathrm{~s}$ time intervals. The charge is then determined from the DC bias voltage required to keep the particle floating. Basically a charged droplet in an environment of relative humidity $\mathrm{RH}_{\mathrm{w}}<1$ cannot evaporate below its terminal radius. Note that without the charge effect, the droplet would have continued to evaporate until it was completely gone. The theoretical fit is the analytical solution to Eq. (5) performed without varying the charge. Basically only the initial slope is fitted, and this slope is used to determine the relative humidity through Eq. (5).

The theoretical prediction of Eq. (4) may be seen as a unique relation between $\mathrm{RH}_{\mathrm{w}}$ and $\sigma_{\mathrm{q}} / T$, which we will now examine. In Fig. 5 we plot this relation along with the measured values of $\mathrm{RH}_{\mathrm{w}}$ and $\sigma_{\mathrm{q}} / T$. The relative accuracy of the DC voltage measurement is best while the particles are still relatively large. However, during the experiment many of the particles undergo Coulomb fission as they pass the Rayleigh instability limit. I.e. due to mutual Coulomb interaction between charges on the droplet surface, the droplet becomes mechanically unstable and breaks up in smaller droplets. Consequently the charge has to be reevaluated in the final state, and this is the main source of the reported uncertainty on $\sigma_{\mathrm{q}}$. Not all the points are within the uncertainty though. These discrepancies are attributed to other experimental uncertainties, including the effect of the electrical fields in the EDB. We do not have good estimates of these possible errors, but since the measurements are generally scattered within the uncertainty around the theoretically predicted line, with a few exceptions of biased low humidity, we assume that these sporadic low biased measurements are caused by some small unknown error in the experiment. We therefore conclude that the theory is confirmed by the experiment. 


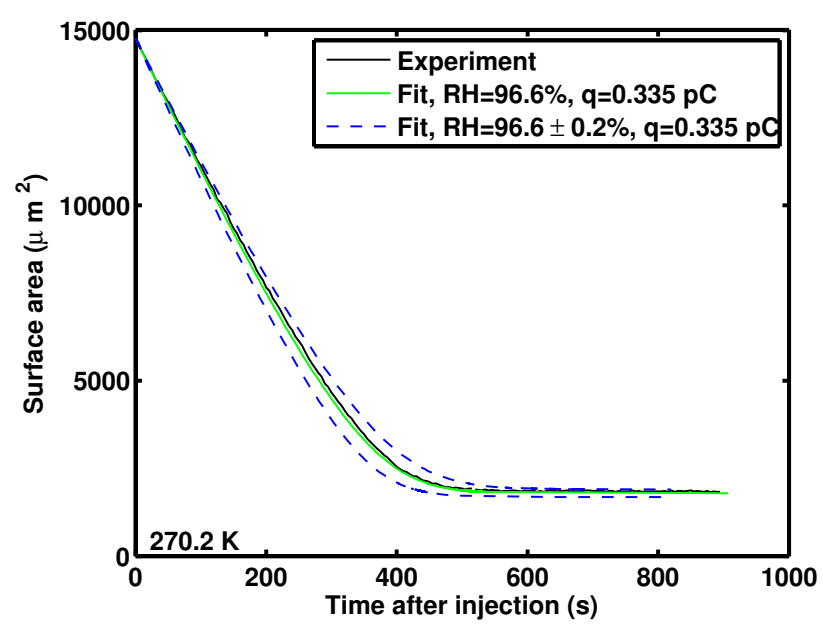

Fig. 4. Example of measured surface area (black curve) of a charged evaporating particle as function of time. The green curve is the analytical solution to Eq. (5). The dashed blue curves illustrate the result of varying the $\mathrm{RH}$ value $\pm 0.2 \%$, which is the estimated uncertainty of the relative humidity.

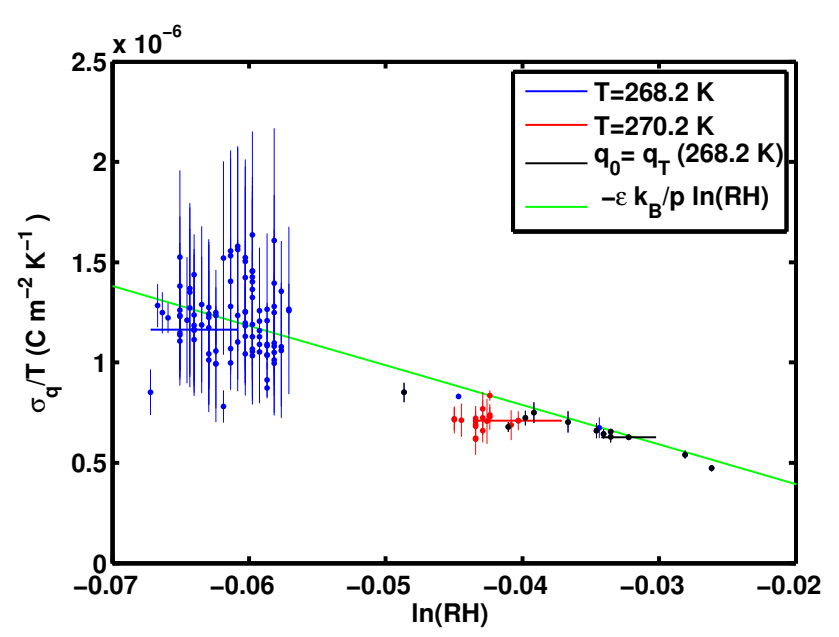

Fig. 5. Surface charge density divided by temperature versus logarithm of relative humidity, for all equilibrated droplets. The vertical lines are error bars showing the instrumental uncertainty, including uncertainty on charge and droplet diameter of each measurement. Blue data: each point represents a droplet equilibrated around $268.2 \mathrm{~K}$. Red data: droplets equilibrated around $270.2 \mathrm{~K}$. Black data: a few droplets at $268.2 \mathrm{~K}$ that were not loosing charge during the experiment, hence the surface charge density is less uncertain in these measurements. The green line shows the theoretical relation between relative humidity and surface charge. Horizontal bars approximate the experimental error on the relative humidity estimated from the slope of the evaporation curves (Fig. 4).

\section{Perspectives}

It may seem a little surprising that the charge-dipole effect has not been observed before, since it is after all a relatively simple experiment, done within the standard operational mode of the EDB. The reason is probably that one has to be aware of the existence of the effect in order not to attribute it to experimental errors or impurities. Moreover, even though the effect is always present in EDB experiments, it is only a small range of RH where it is really clear that the droplets completely fail to evaporate while still visible. As an example Taflin et al. (1988) look at fast evaporation into dry nitrogen where the water vapour pressure is practically zero, or if related to our terminology we could say that $\ln (\mathrm{RH}) \rightarrow-\infty$, i.e. far to the left of our domain in Fig. 5 . Here the surface charge density would diverge, or rather, the droplet is reaching the Rayleigh limit and simply breaks up. Therefore they do not see any stabilization by charge effect in that experiment.

Even though the experiments were done on liquid water droplets we claim that the quantitative predictions and experimental results are applicable for charged ice particles as well, provided that sphericity is maintained. The theory for ice and liquid water are completely identical. Note that the theory is derived solely from gas phase physics. The surface charge cannot have any influence on the bulk phase because the interior electric field is zero. The presence of surface charge could perturb the surface energy, but since the surface effect is completely overruled by the dipole charge interaction we can safely assume that a charge induced perturbation of the surface energy density will still not contribute to the partial pressure of water in either liquid or solid hydrometeors in the size range above one $\mu \mathrm{m}$.

However, the presence of charge will most likely tend to make ice particles less spherical, because features sticking out from an ice surface will tend to hold more electric charge than smoothly rounded areas. Vertices and ends would be preferred by the water dipoles in a condensation process. Seen from a distance of a few particle radii the charged ice particle as a whole will still be subject to an increased water vapor pressure. The only question is how much the geometry will perturb the process. This raises exciting questions about ice particle growth to be examined in future experiments.

This work was inspired by a specific problem, namely the stability of tropical stratospheric ice crystals. As stated in the introduction we are not making any claims about relevance of the charge stabilization effect in tropical stratospheric clouds. We just want to present these laboratory measurements to the atmospheric science community in order to make scientists aware of the charge stabilization mechanism. It is possible that the effect could have an influence on cloud and aerosol processes in other parts of atmospheric science. For example we speculate that the effect could be relevant in thunderclouds, the most obvious place to look for a charge effect. Inside thunderstorms charge densities range from 0 to $10^{9}$ unit 
charges (Bateman et al., 1999). For instance, a hydrometeor of diameter $0.5 \mathrm{~mm}$ and a charge of about $10^{9}$ unit charges would have a surface charge density of $2.5 \times 10^{-4} \mathrm{C} \mathrm{m}^{-2}$. That would decrease the equilibrium relative humidity by $4 \%$, and it would be sufficient to cause a preference for heavily charged hydrometeors compared to neutral hydrometeors in an ongoing condensation processes. This may be thought of as an analog to the Bergeron-Findeisen mechanism. The hydrometeors eventually approach a state of identical surface charge density. The timescale for this process, for a $0.5 \mathrm{~mm}$ droplet, may be estimated from Eq. (5), and is found to be around $20 \mathrm{~h}$, a rather large number. But for a 10 times smaller particle of the same surface charge density the process only takes around ten minutes. So potentially the charge stabilization effect could control, or at least influence, the size distribution in thunderstorm clouds, and in this way influence precipitation. We have not pursued this idea further, and we will just leave these questions open for future research.

Finally we note that it is obvious from our results that the charge stabilization effect should be taken into account in some EDB experiments. Generally, if one measures a parameter which is strongly dependent on the relative humidity, one would have to take into account that the environmental water vapour pressure is smaller than the actual water vapour pressure experienced by a charged droplet. It is straight forward to estimate the severeness of change in water activity from Eq. (4), and include the charge effect accordingly if necessary.

\section{Conclusions}

A theory has been proposed for equilibrium and transient dynamics of charged hydrometeors. The theory's prediction of terminal radius of charged water droplets in a subsaturated environment was verified in an Electrodynamic Balance experiment. The existence of the charge effect is clearly evident from the experiments, and the quantitative theoretical predictions are confirmed reasonably well. The stabilization by charge effect will influence some EDB experiments where the results are sensitive to humidity, and as such it should be taken into account for interpretation of EDB experiments.

\section{Glossary}

$\begin{array}{ll}r & \text { Droplet radius } \\ \rho_{\mathrm{W}} & \text { Liquid water density } \\ R & \text { Gas constant } \\ k_{\mathrm{B}} & \text { Boltzmann's constant } \\ T_{\infty} & \text { Ambient temperature } \\ \mathrm{e}_{\mathrm{s}, 1} & \text { Saturation pressure over plane clean liquid } \\ & \text { water surface } \\ D^{*} & \text { Diffusivity of water in gas phase (corrected } \\ & \begin{array}{l}\text { for kinetic effects) } \\ M_{\mathrm{w}}\end{array} \\ L^{\mathrm{b}} & \text { Mole mass of water } \\ k_{\mathrm{a}}^{*} & \text { Latent heat of evaporation (water) } \\ & \text { Heat conductivity of air (corrected for } \\ q & \text { Particle charge } \\ p & \text { Water dipole moment } \\ \varepsilon_{0} & \text { Vacuum permittivity }\end{array}$

a Murphy and Koop (2005)

b Hall and Pruppacher (1976)

Acknowledgements. This work was supported by the Deutsche Forschungsgemeinschaft within the CAWSES priority program (grant LE 834/2-3), the EC-FP7 project SCOUT-O3 (grant 505390-GOCE-CT-2004), and by the Danish National Research council (FNU), under the project "Cirrus clouds and transport of water vapour in the tropical tropopause layer" (contract number 272-05-0229).

Edited by: J. Curtius

\section{References}

Achtzehn, T., Mueller, R., Duft, D., and Leisner, T.: The Coulomb instability of charged microdroplets, Eur. Phys. J., D34, 311313, doi:10.1140/epjd/e2005-00102-1, 2005.

Bateman, M. G., Marshall, T. C., Stolzenburg, M., and Rust, W. D.: Precipitation charge and size measurements inside a New Mexico mountain thunderstorm, Journal of Geophysical Research (Atmospheres), 104, 9643-9654, doi:10.1029/1998JD200118, 1999.

Chaboureau, J.-P., Cammas, J.-P., Duron, J., Mascart, P. J., Sitnikov, N. M., and Voessing, H.-J.: A numerical study of tropical crosstropopause transport by convective overshoots, Atmos. Chem. Phys., 7, 1731-1740, doi:10.5194/acp-7-1731-2007, 2007.

Cohen, M. D., Flagan, R. C., and Seinfeld, J. H.: Studies of concentrated electrolyte solutions using the electrodynamic balance. 1. Water activities for single-electrolyte solutions, The Journal of Physical Chemistry, 91, 4563-4574, doi:10.1021/j100301a029, 1987.

Corti, T., Luo, B. P., de Reus, M., Brunner, D., Cairo, F., Mahoney, M. J., Martucci, G., Matthey, R., Mitev, V., dos Santos, F. H., Schiller, C., Shur, G., Sitnikov, N. M., Spelten, N., 
Vössing, H. J., Borrmann, S., and Peter, T.: Unprecedented evidence for deep convection hydrating the tropical stratosphere, Geophys. Res. Lett., 35, L10 810, doi:10.1029/2008GL033641, 2008.

Davis, E.: A history of single aerosol particle levitation, Aerosol Science and Technology, 26, 212-254, doi:10.1140/epjd/e200500102-1, 2005.

de Reus, M., Borrmann, S., Bansemer, A., Heymsfield, A. J., Weigel, R., Schiller, C., Mitev, V., Frey, W., Kunkel, D., Krten, A., Curtius, J., Sitnikov, N. M., Ulanovsky, A., and Ravegnani, F.: Evidence for ice particles in the tropical stratosphere from in-situ measurements, Atmos. Chem. Phys., 9, 6775-6792, doi:10.5194/acp-9-6775-2009, 2009.

Duft, D., Achtzehn, T., Müller, R., Huber, B. A., and Leisner, T.: Rayleigh jets from leviated microdroplets, Nature, 421, 128-128, 2003.

Hall, W. W. and Pruppacher, H. R.: The survival of ice particles falling from cirrus clouds in subsaturated air, J. Atmos. Sci., 33, 1995-2006, 1976.

Khaykin, S., Pommereau, J.-P., Korshunov, L., Yushkov, V., Nielsen, J., Larsen, N., Christensen, T., Garnier, A., Lukyanov, A., and Williams, E.: Hydration of the lower stratosphere by ice crystal geysers over land convective systems, Atmos. Chem. Phys., 9, 2275-2287, doi:10.5194/acp-9-2275-2009, 2009.

Koop, T., Luo, B., Tsias, A., and Thomas, P.: Water activity as the determinant for homogeneous ice nucleation in aqueous solutions., Nature, 406, 611-614, 2000.
Lapshin, V. B., Yablolov, M. Y., and Palei, A. A.: Vapor Pressure over a Charged Drop, Russian J. Phys. Chem., 76, 1727-1729, 2002.

Murphy, D. M. and Koop, T.: Review of the vapour pressures of ice and supercooled water for atmospheric applications, Q. J. R. Meteorological Society, 131, 1539-1565, 2005.

Nielsen, J. K., Larsen, N., Cairo, F., Di Donfrancesco, G., Rosen, J. M., Durry, G., Held, G., and Pommereau, J. P.: Solid particles in the tropical lowest stratosphere, Atmos. Chem. Phys., 7, 685695, doi:10.5194/acp-7-685-2007, 2007.

Pruppacher, H. and Klett, J.: Microphysics of Clouds and Precipitation, vol. 18 of Atmospheric and Oceanographic Sciences Library, Springer, second revised and enlarged edition with an introduction to cloud chemistry and cloud electricity, 170-172, 509-511, 1997.

Rayleigh, L.: On the equilibrium of liquid conducting masses charged with electricity, Philosphical Magazine, 14, 184-186, 1882.

Rusanov, A. I.: Thermodynamic theory of nucleation on charged particles, Journal of Colloid and Interface Science, 68, 32-47, doi:10.1016/0021-9797(79)90256-X, 1979.

Taflin, D. C., Zhang, S. H., Allen, T., and Davis, E. J.: Measurement of Droplet Interfacial Phenomena by Light-Scattering Techniques, Aiche J., 34, 1310-1320, 1988.

Thomson, J. J. and Thomson, G. P.: Conduction of Electricity through Gases, 1, 310-337, Cambridge University Press, Cambridge, 1928. 